Marquette University

e-Publications@Marquette

Electrical and Computer Engineering Faculty

Electrical and Computer Engineering, Department

Research and Publications

$1-1-2016$

\title{
Robust Multi-Criteria Optimal Fuzzy Control of Discrete-Time Nonlinear Systems
}

Xin Wang

Marquette University

Edwin E. Yaz

Marquette University, edwin.yaz@marquette.edu

Published version. Systems Science \& Control Engineering, Vol. 4, No. 1 (2016): 31-38. DOI. (C) 2016

Taylor \& Francis. Used with permission. 


\title{
Systems Science \& Control Engineering
}

An Open Access Journal

ISSN: (Print) 2164-2583 (Online) Journal homepage: http://www.tandfonline.com/loi/tssc20

\section{Robust multi-criteria optimal fuzzy control of discrete-time nonlinear systems}

\author{
Xin Wang \& Edwin E. Yaz
}

To cite this article: Xin Wang \& Edwin E. Yaz (2016) Robust multi-criteria optimal fuzzy control of discrete-time nonlinear systems, Systems Science \& Control Engineering, 4:1, 31-38, DOI: 10.1080/21642583.2016.1138338

To link to this article: http://dx.doi.org/10.1080/21642583.2016.1138338

\section{(c) 2016 The Author(s). Published by Taylor \&
Francis}

Accepted author version posted online: 12
Jan 2016.
Published online: 05 Feb 2016.
Submit your article to this journal

Џ Article views: 410

Q View related articles $\longleftarrow$

View Crossmark data $\nearrow$ 


\title{
Robust multi-criteria optimal fuzzy control of discrete-time nonlinear systems
}

\author{
Xin Wang ${ }^{\mathrm{a}}$ and Edwin E. Yaz ${ }^{\mathrm{b}}$ \\ ${ }^{a}$ Department of Electrical and Computer Engineering, Southern Illinois University Edwardsville, Edwardsville, IL, USA; ${ }^{\mathrm{b}}$ Electrical and Computer \\ Engineering Department, Marquette University, Milwaukee, WI, USA
}

\begin{abstract}
This paper presents a novel fuzzy control design of discrete-time nonlinear systems with multiple performance criteria. The purpose behind this work is to improve the traditional fuzzy controller performance to satisfy several performance criteria simultaneously to secure quadratic optimality with an inherent stability property together with a dissipativity type of disturbance reduction. The Takagi-Sugeno-type fuzzy model is used in our control system design. By solving a linear matrix inequality at each time step, the optimal control solution can be found to satisfy mixed performance criteria. The effectiveness of the proposed technique is demonstrated by simulation of the control of the inverted pendulum system on a cart.
\end{abstract}

\section{ARTICLE HISTORY}

Received 12 December 2015 Accepted 2 January 2016

\section{KEYWORDS}

Fuzzy logic; robust control; linear matrix inequality

\section{Introduction}

Fuzzy control systems have recently shown growing popularity in nonlinear system control applications (Takagi \& Sugeno, 1985; Tanaka \& Sugeno, 1990; Tanaka, Ikeda, \& Wang, 1996; Tanaka \& Wang, 2001; Wang, 1994; Wang, Tanaka, \& Griffin, 1996). A fuzzy control system is essentially an effective way to decompose the task of nonlinear system control into a group of local linear controls based on a set of design-specific model rules. Fuzzy control also provides a mechanism to blend these local linear control problems all together to achieve overall control of the original nonlinear system. In this regard, a fuzzy control technique has its unique advantage over other kinds of nonlinear control techniques. Latest research on fuzzy control systems design is aimed to improve the optimality and robustness of the controller performance by combining the advantage of modern control theory with the Takagi-Sugeno fuzzy model (Takagi \& Sugeno, 1985; Tanaka \& Sugeno, 1990; Tanaka \& Wang, 2001; Tanaka et al., 1996; Wang, 1994; Wang et al., 1996).

In this paper, we address the nonlinear state feedback control design of discrete-time nonlinear fuzzy control systems using the linear matrix inequality (LMI) approach. We characterize the solution of the nonlinear discretetime control problem with the LMI, which provides a sufficient condition for satisfying various performance criteria. A preliminary investigation into the LMI approach to nonlinear fuzzy control systems can be found in Takagi and Sugeno (1985), Tanaka and Sugeno (1990), and
Wang (1994). The purpose behind this novel approach is to convert a nonlinear system control problem into a convex optimization problem which is solved by an $\mathrm{LMI}$ at each time step. The recent development in convex optimization provides efficient algorithms for solving LMIs. If a solution can be expressed in an LMI form, then there exist optimization algorithms providing efficient global numerical solutions (Boyd, Ghaoui, Feron, \& Balakrishnan, 1994). Therefore if the LMI is feasible, then the LMI control technique provides globally stable solutions satisfying the corresponding mixed performance criteria at each time step (Huang \& Lu, 1996; Mohseni, Yaz, \& Olejniczak, 1998; Wang \& Yaz, 2009; Wang \& Yaz, 2010, July; Wang and Yaz, 2010, December; Wang, Yaz, \& Jeong, 2010, July; Wang, Yaz, \& Yaz, 2010, December; Wang, Yaz, \& Yaz, 2011). We further propose to employ mixed performance criteria to design the controller guaranteeing the quadratic sub-optimality with an inherent stability property in combination with a dissipativity type of disturbance attenuation.

In the following section, we first describe the TakagiSugeno fuzzy model. We then introduce the mixed performance criteria in Section 3. Then, the LMI control solution is derived to characterize the optimal and robust fuzzy control of nonlinear systems. Finally, an inverted pendulum on a cart control problem is used as an illustrative example. The following notation is used in this work: $x \in \Re^{n}$ denotes $n$-dimensional real vector with norm $\|x\|=\left(x^{\top} x\right)^{1 / 2}$, where $(\cdot)^{\top}$ indicates transpose. $A \geq 0$ for a

\section{CONTACT Xin Wang xwang@siue.edu}


symmetric matrix denotes a positive semi-definite matrix. $I_{2}$ is the space of infinite sequences of finite dimensional random vectors with finite energy: $\sum_{k=0}^{\infty}\left\|x_{k}\right\|^{2}<\infty$.

\section{Takagi-Sugeno system model}

The importance of the Takagi-Sugeno fuzzy system model is that it provides an effective way to decompose a complicated nonlinear system into local dynamical relations and express those local dynamics of each fuzzy implication rule by a linear system model. The overall fuzzy nonlinear system model is achieved by fuzzy 'blending' of the linear system models, so that the overall nonlinear control performance is achieved.

At time step $k$, the ith rule of the Takagi-Sugeno fuzzy model can be expressed by the following forms:

Model rule $i$ :

IF $\varphi_{1}(k)$ is $M_{i 1}, \varphi_{2}(k)$ is $M_{i 2}, \ldots$, and $\varphi_{p}(k)$ is $M_{i p}$,

THEN, the input-affine discrete-time fuzzy system equation is

$$
\begin{aligned}
& \left\{\begin{array}{l}
x(k+1)=A_{i} x(k)+B_{i} u(k)+F_{i} w(k) \\
y(k)=C_{i} x(k)+D_{i} u(k)+Z_{i} w(k)
\end{array}\right. \\
& i=1,2,3, \ldots, r,
\end{aligned}
$$

where

$x(k) \in \Re^{n}$ - state vector,

$u(k) \in \Re^{m}$ - control input vector,

$y(k) \in \Re^{q}$ - performance output vector,

$w(k) \in \Re^{s}-I_{2}$ type of disturbance,

$r$ - total number of the model rules,

$M_{i j}$ - fuzzy set,

$A_{i} \in \Re^{n \times n}, B_{i} \in \Re^{n \times m}, F_{i} \in \Re^{n \times s}, C_{i} \in \Re^{q \times n}, D_{i} \in \Re^{q \times m}$, $Z_{i} \in \Re^{q \times s}$ - coefficient matrices,

$\varphi_{1}, \ldots, \varphi_{p}-$ known premise variables which can be functions of state variables, external disturbance and time.

It is assumed that the premises are not the function of the input vector $u(k)$, which is needed to avoid the defuzzification process of fuzzy controller. If we use $\varphi(k)$ to denote the vector containing all the individual elements $\varphi_{1}(k), \ldots, \varphi_{p}(k)$, then the overall fuzzy system is

$$
\begin{aligned}
x(k+1) & =\frac{\sum_{i=1}^{r} g_{i}(\varphi(k))\left\{A_{i} x(k)+B_{i} u(k)+F_{i} w(k)\right\}}{\sum_{i=1}^{r} g_{i}(\varphi(k))} \\
& =\sum_{i=1}^{r} h_{i}(\varphi(k))\left\{A_{i} x(k)+B_{i} u(k)+F_{i} w(k)\right\}, \\
y(k) & =\frac{\sum_{i=1}^{r} g_{i}(\varphi(k))\left\{C_{i} x(k)+D_{i} u(k)+Z_{i} w(k)\right\}}{\sum_{i=1}^{r} g_{i}(\varphi(k))} \\
& =\sum_{i=1}^{r} h_{i}(\varphi(k))\left\{C_{i} x(k)+D_{i} u(k)+Z_{i} w(k)\right\},
\end{aligned}
$$

where

$$
\begin{aligned}
\varphi(k) & =\left[\varphi_{1}(k), \varphi_{2}(k), \ldots, \varphi_{p}(k)\right], \\
g_{i}(\varphi(k)) & =\prod_{j=1}^{p} M_{i j}\left(\varphi_{j}(k)\right), \\
h_{i}(\varphi(k)) & =\frac{g_{i}(\varphi(k))}{\sum_{i=1}^{r} g_{i}(\varphi(k))},
\end{aligned}
$$

for all $k$. The term $M_{i j}\left(\varphi_{j}(k)\right)$ is the grade membership of $\varphi_{j}(k)$ in $M_{i j}$. Since

$$
\left\{\begin{array}{l}
\sum_{i=1}^{r} g_{i}(\varphi(k))>0 \\
g_{i}(\varphi(k)) \geq 0, \quad i=1,2,3, \ldots, r
\end{array}\right.
$$

we have

$$
\left\{\begin{array}{l}
\sum_{i=1}^{r} h_{i}(\varphi(k))=1 \\
h_{i}(\varphi(k)) \geq 0, \quad i=1,2,3, \ldots, r
\end{array}\right.
$$

for all $k$.

It is assumed that the state feedback is available and the nonlinear state feedback control input is given by

$$
u(k)=-\sum_{i=1}^{r} h_{i}(\varphi(k)) K_{i} x(k) .
$$

Substituting this into the system and performance output equation, we have

$$
\begin{aligned}
x(k+1)= & \sum_{i=1}^{r} \sum_{j=1}^{r} h_{i}(\varphi(k)) h_{j}(\varphi(k))\left\{A_{i}-B_{i} K_{j}\right\} x(k) \\
& +\sum_{i=1}^{r} h_{i}(\varphi(k)) F_{i} w(k), \\
y(k)= & \sum_{i=1}^{r} \sum_{j=1}^{r} h_{i}(\varphi(k)) h_{j}(\varphi(k))\left\{C_{i}-D_{i} K_{j}\right\} x(k) \\
& +\sum_{i=1}^{r} h_{i}(\varphi(k)) Z_{i} w(k) .
\end{aligned}
$$

Using the notation

$$
\begin{aligned}
& G_{i j}=A_{i}-B_{i} K_{j}, \\
& H_{i j}=C_{i}-D_{i} K_{j},
\end{aligned}
$$

the system equation becomes

$$
\begin{aligned}
x(k+1)= & \sum_{i=1}^{r} \sum_{j=1}^{r} h_{i}(\varphi(k)) h_{j}(\varphi(k)) \cdot G_{i j} \cdot x(k) \\
& +\sum_{i=1}^{r} h_{i}(\varphi(k)) F_{i} w(k)
\end{aligned}
$$




$$
\begin{aligned}
y(k)= & \sum_{i=1}^{r} \sum_{j=1}^{r} h_{i}(\varphi(k)) h_{j}(\varphi(k)) \cdot H_{i j} \cdot x(k) \\
& +\sum_{i=1}^{r} h_{i}(\varphi(k)) Z_{i} w(k) .
\end{aligned}
$$

\section{General performance criteria}

Consider the quadratic Lyapunov function

$$
V(k)=x^{\top}(k) P(k) x(k)>0,
$$

for the following difference inequality:

$$
\begin{aligned}
& V(k+1)-V(k)+x^{\top}(k) Q x(k)+u^{\top}(k) R u(k) \\
& \quad+\alpha \cdot y^{\top}(k) y(k)-\beta \cdot y^{\top}(k) w(k)+\gamma \cdot w^{\top}(k) w(k) \leq 0,
\end{aligned}
$$

with $Q>0, R>0$ functions of $x(k)$.

Note that upon summation over $k$, Equation (17) yields

$$
V(N)+\sum_{k=0}^{N-1}\left[\begin{array}{c}
x^{\top}(k) Q(k) x(k)+u^{\top}(k) R(k) u(k) \\
+\alpha \cdot y^{\top}(k) y(k)-\beta \cdot y^{\top}(k) w(k) \\
+\gamma \cdot w^{\top}(k) w(k)
\end{array}\right] \leq V(0) .
$$

By properly specifying the value of weighing matrices $Q, R, C_{i}, D_{i}, Z_{i}$ and $\alpha, \beta, \gamma$, mixed performance criteria can be used in nonlinear control design, which yields a mixed nonlinear quadratic regulator (NLQR) in combination with the dissipativity-type performance index with disturbance reduction capability. For example, if we take $\alpha=1, \beta=0, \gamma<0$, Equation (18) yields

$$
\begin{aligned}
& V(N)+\sum_{k=0}^{N-1}\left[x^{\top}(k) Q(k) x(k)+u^{\top}(k) R(k) u(k)\right. \\
& \left.+y^{\top}(k) y(k)\right] \leq V(0)-\gamma \cdot \sum_{k=0}^{N-1} w^{\top}(k) w(k),
\end{aligned}
$$

which is a mixed NLQR- $H_{\infty}$ design (Wang \& Yaz, 2009, 2010, 2011). In Equation (19), $\gamma$ can be minimized to achieve a smaller $I_{2}-I_{2}$ or $H_{\infty}$ gain for the closed-loop system (Basar \& Bernhard, 1995; Van der Shaft, 1993).

Other possible performance criteria which can be used in this framework with various design parameters $\alpha, \beta, \gamma$ are given in Table 1. Design coefficients $\alpha$ and $\gamma$ can be maximized or minimized to optimize the controller behaviour. It should also be noted that the satisfaction of any of the criteria in Table 1 will also guarantee asymptotic stability of the controlled system (Khalil, 2002; Vidyasagar, 2002).
Table 1. Various performance criteria in a general framework.

\begin{tabular}{llrl}
\hline$\alpha$ & $\beta$ & $\gamma$ & \multicolumn{1}{c}{ Performance criteria } \\
\hline 1 & 0 & $<0$ & NLQR- $H_{\infty}$ design \\
0 & 1 & 0 & NLQR-passivity design \\
0 & 1 & $>0$ & NLQR-input strict passivity design \\
$>0$ & 1 & 0 & NLQR-output strict passivity design \\
$>0$ & 1 & $>0$ & NLQR-very strict passivity \\
\hline
\end{tabular}

\section{Fuzzy LMI control with general performance criteria}

The following theorem summarizes the main results of the paper:

Theorem 1: Given the closed-loop system (10), performance output (11), if there exist matrices $S=P^{-1}>0$ for all $k \geq 0$, such that the following LMI holds:

$$
\left[\begin{array}{cccccc}
\Xi_{11} & \Xi_{12} & \Xi_{13} & \Xi_{14} & \Xi_{15} & \Xi_{16} \\
* & \Xi_{22} & \Xi_{23} \Xi_{24} & 0 & 0 \\
* & * & S & 0 & 0 & 0 \\
* & * & * & I & 0 & 0 \\
* & * & * & * & R^{-1} & 0 \\
* & * & * & * & * & l
\end{array}\right] \geq 0
$$

where

$$
\begin{aligned}
& \Xi_{11}=S, \\
& \Xi_{12}=\frac{\beta}{4}\left[C_{i} S-D_{i} Y_{j}+C_{j} S-D_{j} Y_{i}\right]^{\top}, \\
& \Xi_{13}=\frac{1}{2}\left[A_{i} S-B_{i} Y_{j}+A_{j} S-B_{j} Y_{i}\right]^{\top}, \\
& \Xi_{14}=\frac{1}{2} \alpha^{1 / 2} \cdot\left[C_{i} S-D_{i} Y_{j}+C_{j} S-D_{j} Y_{j}\right]^{\top}, \\
& \Xi_{15}=\frac{1}{2}\left[Y_{i}+Y_{j}\right]^{\top}, \\
& \Xi_{16}=S Q^{\top / 2}, \\
& \Xi_{22}=-\gamma I+\frac{\beta}{2}\left(Z_{i}+Z_{j}\right)^{\top}, \\
& \Xi_{23}=\frac{1}{2} \alpha^{1 / 2} \cdot\left(F_{i}+F_{j}\right)^{\top}, \\
& \Xi_{24}=\frac{1}{2} \alpha^{1 / 2} \cdot\left[Z_{i}+Z_{j}\right]^{\top},
\end{aligned}
$$

and

$$
S(k+1)>S(k),
$$

where $S(k)=P^{-1}(k)$, then Equation (18) is satisfied with the feedback control gain being found by

$$
K_{i}(k)=Y_{i}(k) \cdot P(k) \quad \text { and } \quad K_{j}(k)=Y_{j}(k) \cdot P(k) .
$$


Proof: The performance index inequality (18) can be explicitly written as

$$
\begin{aligned}
& {\left[\sum_{i=1}^{r} \sum_{j=1}^{r} h_{i}(\phi(k)) h_{j}(\phi(k)) \cdot G_{i j} \cdot x(k)\right]^{\top}} \\
& +\sum_{i=1}^{r} h_{i}(\phi(k)) F_{i} w(k) \\
& \cdot P \cdot\left[\begin{array}{c}
\sum_{i=1}^{r} \sum_{j=1}^{r} h_{i}(\phi(k)) h_{j}(\phi(k)) \cdot G_{i j} \cdot x(k) \\
+\sum_{i=1}^{r} h_{i}(\phi(k)) F_{i} w(k)
\end{array}\right] \\
& -x^{\top}(k) P x(k)+x^{\top}(k) Q x(k) \\
& +\left[-\sum_{i=1}^{r} h_{i}(\phi(k)) K_{i} x(k)\right]^{\top} R\left[-\sum_{i=1}^{r} h_{i}(\phi(k)) K_{i} x(k)\right] \\
& +\alpha\left[\begin{array}{c}
\sum_{i=1}^{r} \sum_{j=1}^{r} h_{i}(\phi(k)) h_{j}(\phi(k)) \cdot H_{i j} \cdot x(k) \\
+\sum_{i=1}^{r} h_{i}(\phi(k)) Z_{i} w(k)
\end{array}\right]^{\top} \\
& \cdot\left[\begin{array}{c}
\sum_{i=1}^{r} \sum_{j=1}^{r} h_{i}(\phi(k)) h_{j}(\phi(k)) \cdot H_{i j} \cdot x(k) \\
+\sum_{i=1}^{r} h_{i}(\phi(k)) Z_{i} w(k)
\end{array}\right] \\
& -\beta\left[\begin{array}{c}
\sum_{i=1}^{r} \sum_{j=1}^{r} h_{i}(\phi(k)) h_{j}(\phi(k)) \cdot H_{i j} \cdot x(k) \\
+\sum_{i=1}^{r} h_{i}(\phi(k)) Z_{i} w(k)
\end{array}\right]^{\top} \\
& w(k)+\gamma w^{\top}(k) w(k) \leq 0 .
\end{aligned}
$$

Equivalently,

$$
\begin{aligned}
& {\left[x^{\top}(k) \quad w^{\top}(k)\right]\left[\begin{array}{cc}
-P+Q & 0 \\
0 & \gamma l
\end{array}\right]\left[\begin{array}{l}
x(k) \\
w(k)
\end{array}\right]} \\
& +\left[\begin{array}{ll}
x^{\top}(k) & w^{\top}(k)
\end{array}\right]\left[\begin{array}{ll}
\left(\sum_{i} \sum_{j} h_{i} h_{j} G_{i j}\right) & \left(\sum_{i} h_{i} F_{i}\right)
\end{array}\right]^{\top} \\
& \text {.P. }\left[\begin{array}{ll}
\left(\sum_{i} \sum_{j} h_{i} h_{j} G_{i j}\right) & \left.\left(\sum_{i} h_{i} F_{i}\right)\right]
\end{array}\right]\left[\begin{array}{l}
x(k) \\
w(k)
\end{array}\right] \\
& +x^{\top}(k)\left[\sum_{i} h_{i} K_{i}\right]^{\top} R\left[\sum_{i} h_{i} K_{i}\right] x(k) \\
& +\alpha \cdot\left[\begin{array}{ll}
x^{\top}(k) & w^{\top}(k)
\end{array}\right]\left[\begin{array}{ll}
\sum_{i} \sum_{j} h_{i} h_{j} H_{i j} & \sum_{i} h_{i} Z_{i}
\end{array}\right]^{\top} \\
& \cdot\left[\begin{array}{ll}
\sum_{i} \sum_{j} h_{i} h_{j} H_{i j} & \sum_{i} h_{i} Z_{i}
\end{array}\right]\left[\begin{array}{l}
x(k) \\
w(k)
\end{array}\right] \\
& -\beta \cdot\left[\begin{array}{ll}
x^{\top}(k) & w^{\top}(k)
\end{array}\right]\left[\begin{array}{ll}
\sum_{i} \sum_{j} h_{i} h_{j} H_{i j} & \sum_{i} h_{i} Z_{i}
\end{array}\right]^{\top} \\
& w(k) \leq 0,
\end{aligned}
$$

which can be written, after collecting terms, as

$$
\begin{aligned}
& {\left[\begin{array}{ll}
x^{\top}(k) & w^{\top}(k)
\end{array}\right]\left[\begin{array}{cc}
\Upsilon_{11} & \Upsilon_{12} \\
* & \Upsilon_{22}
\end{array}\right]\left[\begin{array}{l}
x(k) \\
w(k)
\end{array}\right]} \\
& -\left[\begin{array}{ll}
x^{\top}(k) & w^{\top}(k)
\end{array}\right]\left[\begin{array}{ll}
\left(\sum_{i} \sum_{j} h_{i} h_{j} G_{i j}\right) & \left(\sum_{i} h_{i} F_{i}\right)
\end{array}\right]^{\top} \\
& \text {.P. }\left[\begin{array}{ll}
\left(\sum_{i} \sum_{j} h_{i} h_{j} G_{i j}\right) & \left.\left(\sum_{i} h_{i} F_{i}\right)\right]
\end{array}\right]\left[\begin{array}{l}
x(k) \\
w(k)
\end{array}\right] \\
& -\alpha \cdot\left[\begin{array}{ll}
x^{\top}(k) & w^{\top}(k)
\end{array}\right]\left[\begin{array}{ll}
\sum_{i} \sum_{j} h_{i} h_{j} H_{i j} & \sum_{i} h_{i} Z_{i}
\end{array}\right]^{\top} \\
& \cdot\left[\sum_{i} \sum_{j} h_{i} h_{j} H_{i j} \quad \sum_{i} h_{i} Z_{i}\right]\left[\begin{array}{l}
x(k) \\
w(k)
\end{array}\right] \geq 0 \text {, }
\end{aligned}
$$

where

$$
\begin{aligned}
& \Upsilon_{11}=P-Q-\left[\sum_{i} h_{i} K_{i}\right]^{\top} R\left[\sum_{i} h_{i} K_{i}\right], \\
& \Upsilon_{12}=\frac{\beta}{2}\left[\sum_{i} \sum_{j} h_{i} h_{j} H_{i j}\right]^{\top}, \\
& \Upsilon_{22}=-\gamma I+\beta\left[\sum_{i} h_{i} Z_{i}\right]^{\top} .
\end{aligned}
$$

Equivalently, we have

$$
\begin{aligned}
& {\left[\begin{array}{cc}
\Upsilon_{11} & \Upsilon_{12} \\
* & \Upsilon_{22}
\end{array}\right]-\left[\left(\sum_{i} \sum_{j} h_{i} h_{j} G_{i j}\right)\left(\sum_{i} h_{i} F_{i}\right)\right]^{\top}} \\
& . P \cdot\left[\left(\sum_{i} \sum_{j} h_{i} h_{j} G_{i j}\right)\left(\sum_{i} h_{i} F_{i}\right)\right] \\
& -\alpha \cdot\left[\begin{array}{ll}
\sum_{i} \sum_{j} h_{i} h_{j} H_{i j} & \sum_{i} h_{i} Z_{i}
\end{array}\right]^{\top} \\
& \cdot\left[\begin{array}{ll}
\sum_{i} \sum_{j} h_{i} h_{j} H_{i j} & \sum_{i} h_{i} Z_{i}
\end{array}\right] \geq 0 .
\end{aligned}
$$

By applying the Schur complement, we obtain

$$
\begin{gathered}
{\left[\begin{array}{ccc}
\Upsilon_{11} & \Upsilon_{12} & \left(\sum_{i} \sum_{j} h_{i} h_{j} G_{i j}\right)^{\top} \\
* & \Upsilon_{22} & \left(\sum_{i} h_{i} F_{i}\right)^{\top} \\
* & * & P^{-1}
\end{array}\right]^{\top}} \\
-\alpha \cdot\left[\begin{array}{lll}
\sum_{i} \sum_{j} h_{i} h_{j} H_{i j} & \sum_{i} h_{i} Z_{i}
\end{array}\right]^{\top} \\
\cdot\left[\begin{array}{ccc}
\sum_{i} \sum_{j} h_{i} h_{j} H_{i j} & \sum_{i} h_{i} Z_{i}
\end{array}\right] \geq 0 .
\end{gathered}
$$


By applying the Schur complement again, we obtain

$$
\left[\begin{array}{cccc}
\Upsilon_{11} & \Upsilon_{12} & \left(\sum_{i} \sum_{j} h_{i} h_{j} G_{i j}\right)^{\top} & \alpha^{1 / 2}\left(\sum_{i} \sum_{j} h_{i} h_{j} H_{i j}\right)^{\top} \\
* & \Upsilon_{22} & \left(\sum_{i} h_{i} F_{i}\right)^{\top} & \alpha^{1 / 2}\left(\sum_{i} h_{i} Z_{i}\right)^{\top} \\
* & * & P^{-1} & 0 \\
* & * & * & l
\end{array}\right]
$$$$
\geq 0 \text {. }
$$

Equivalently, the following inequality holds:

$$
\begin{aligned}
& {\left[\begin{array}{cccc}
\Psi_{11} & \Psi_{12} & \left(\sum_{i} \sum_{j} h_{i} h_{j} G_{i j}\right)^{\top} & \alpha^{1 / 2}\left(\sum_{i} \sum_{j} h_{i} h_{j} H_{i j}\right)^{\top} \\
* & \Psi_{22} & \left(\sum_{i} h_{i} F_{i}\right)^{\top} & \alpha^{1 / 2}\left(\sum_{i} h_{i} Z_{i}\right)^{\top} \\
* & * & P^{-1} & 0 \\
* & * & * & l
\end{array}\right]} \\
& -\left[\begin{array}{c}
\left(\sum_{i} h_{i} K_{i}\right)^{\top} \\
0 \\
0 \\
0
\end{array}\right] R\left[\left(\sum_{i} h_{i} K_{i}\right) \quad 0 \quad 000\right] \geq 0
\end{aligned}
$$

where

$$
\begin{aligned}
& \Psi_{11}=P-Q, \\
& \Psi_{12}=\frac{\beta}{2}\left[\sum_{i} \sum_{j} h_{i} h_{j} H_{i j}\right]^{\top}, \\
& \Psi_{22}=-\gamma I+\beta\left[\sum_{i} h_{i} Z_{i}\right]^{\top} .
\end{aligned}
$$
have

By applying the Schur complement one more time, we

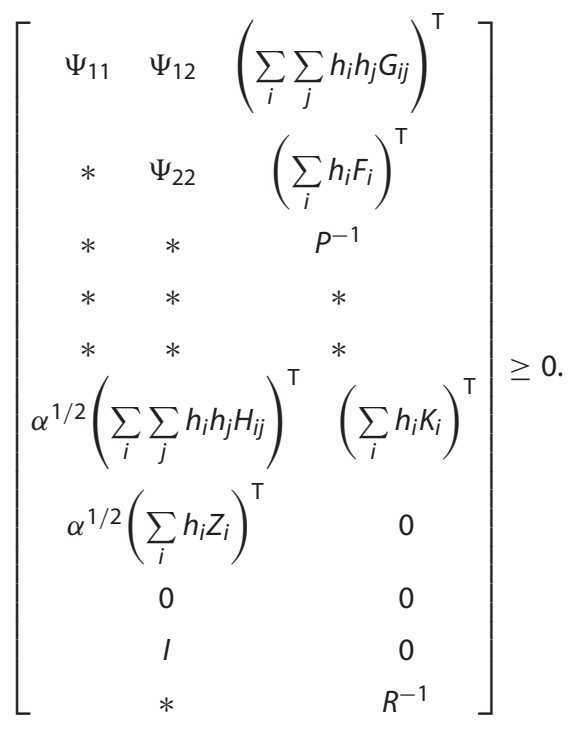

By factoring out the $\sum_{i} \sum_{j} h_{i}\left(\varphi_{k}\right) h_{j}\left(\varphi_{k}\right)$ term, we have

$$
\left[\begin{array}{ccccc}
\Omega_{11} & \Omega_{12} & \Omega_{13} & \Omega_{14} & \Omega_{15} \\
* & \Omega_{22} & \Omega_{23} & \Omega_{24} & 0 \\
* & * & P^{-1} & 0 & 0 \\
* & * & * & I & 0 \\
* & * & * & * & R^{-1}
\end{array}\right] \geq 0,
$$

$$
\begin{aligned}
& \Omega_{11}=P-Q, \\
& \Omega_{12}=\frac{\beta}{4}\left[H_{i j}+H_{j i}\right]^{\top}, \\
& \Omega_{13}=\frac{1}{2}\left[G_{i j}+G_{j i}\right]^{\top}, \\
& \Omega_{14}=\frac{1}{2} \alpha^{1 / 2} \cdot\left[H_{i j}+H_{j i}\right]^{\top}, \\
& \Omega_{15}=\frac{1}{2}\left[K_{i}+K_{j}\right]^{\top}, \\
& \Omega_{22}=-\gamma I+\frac{\beta}{2}\left(Z_{i}+Z_{j}\right)^{\top}, \\
& \Omega_{23}=\frac{1}{2}\left(F_{i}+F_{j}\right)^{\top}, \\
& \Omega_{24}=\frac{1}{2} \alpha^{1 / 2} \cdot\left[Z_{i}+Z_{j}\right]^{\top} .
\end{aligned}
$$

By pre-multiplying and post-multiplying the matrix with the block diagonal matrix block diag $\{S, I, I, I, I\}$, where $S=P^{-1}$, and applying the Schur complement again, the following $L M I$ result is obtained:

$$
\left[\begin{array}{cccccc}
\Xi_{11} & \Xi_{12} & \Xi_{13} & \Xi_{14} & \Xi_{15} & \Xi_{16} \\
* & \Xi_{22} & \Xi_{23} & \Xi_{24} & 0 & 0 \\
* & * & S & 0 & 0 & 0 \\
* & * & * & I & 0 & 0 \\
* & * & * & * & R^{-1} & 0 \\
* & * & * & * & * & l
\end{array}\right] \geq 0
$$

where

$$
\begin{aligned}
& \Xi_{11}=S, \\
& \Xi_{12}=\frac{\beta}{4}\left[C_{i} S-D_{i} Y_{j}+C_{j} S-D_{j} Y_{i}\right]^{\top}, \\
& \Xi_{13}=\frac{1}{2}\left[A_{j} S-B_{i} Y_{j}+A_{j} S-B_{j} Y_{i}\right]^{\top}, \\
& \Xi_{14}=\frac{1}{2} \alpha^{1 / 2} \cdot\left[C_{i} S-D_{i} Y_{j}+C_{j} S-D_{j} Y_{i}\right]^{\top}, \\
& \Xi_{15}=\frac{1}{2}\left[Y_{i}+Y_{j}\right]^{\top}, \\
& \Xi_{16}=S Q^{\top / 2}, \\
& \Xi_{22}=-\gamma I+\frac{\beta}{2}\left(Z_{i}+Z_{j}\right)^{\top},
\end{aligned}
$$




$$
\begin{aligned}
& \Xi_{23}=\frac{1}{2} \alpha^{1 / 2} \cdot\left(F_{i}+F_{j}\right)^{\top}, \\
& \Xi_{24}=\frac{1}{2} \alpha^{1 / 2} \cdot\left[Z_{i}+Z_{j}\right]^{\top},
\end{aligned}
$$

where $S=P^{-1}$ and feedback gain $K_{i}(k)=Y_{i}(k) \cdot P(k)$ and $K_{j}(k)=Y_{j}(k) \cdot P(k)$.

Hence, if the LMI (36) holds, performance criteria inequality (18) is satisfied. This concludes the proof of theorem.

\section{Application to the inverted pendulum on a cart}

The inverted pendulum on a cart problem is a benchmark control problem used widely to test control algorithms. A pendulum beam attached at one end can rotate freely in the vertical two-dimensional plane. The angle of the beam with respect to the vertical direction is denoted at angle $\theta$. The external force $u$ is desired to set angle of the beam $\theta$ and angular velocity $\dot{\theta}$ to zero while satisfying the mixed performance criteria. A model of the inverted pendulum on a cart problem is given by Baumann and Rugh (1986) and Tanaka and Wang (2001):

$$
\left\{\begin{aligned}
\dot{x}_{1} & =x_{2}+\varepsilon_{1} \cdot w \\
\dot{x}_{2} & =\frac{g \sin \left(x_{1}\right)-a m L x_{2}^{2} \cdot \sin \left(2 x_{1}\right) / 2-a \cos \left(x_{1}\right) u}{4 L / 3-a m L \cos ^{2}\left(x_{1}\right)} \\
& +\varepsilon_{2} \cdot w
\end{aligned}\right.
$$

where

$x_{1}$ - angle of the pendulum from the vertical direction,

$x_{2}$ - angular velocity of the pendulum,

$g$ - gravity constant,

$m$ - mass of the pendulum,

$M$ - mass of the cart,

$L$ - length to the pendulum centre of mass. The total length of pendulum equals $2 L$,

$u$ - external force, control input to the system,

$w-L_{2}$ type of disturbance,

$a$ - constant $a=1 /(m+M)$,

$\varepsilon_{1}, \varepsilon_{2}$ - weighting coefficients of the disturbance.

Due to the system nonlinearity, we approximate the system using the following two-rule fuzzy model:

Rule 1:

IF $\left|x_{1}(k)\right|$ is close to zero,

THEN $x(k+1)=A_{1} x(k)+B_{1} u(k)+F_{1} w(k)$.

Rule 2:

IF $\left|x_{1}(k)\right|$ is close to $\pi / 2$,

$\operatorname{THEN} x(k+1)=A_{2} x(k)+B_{2} u(k)+F_{2} w(k)$,

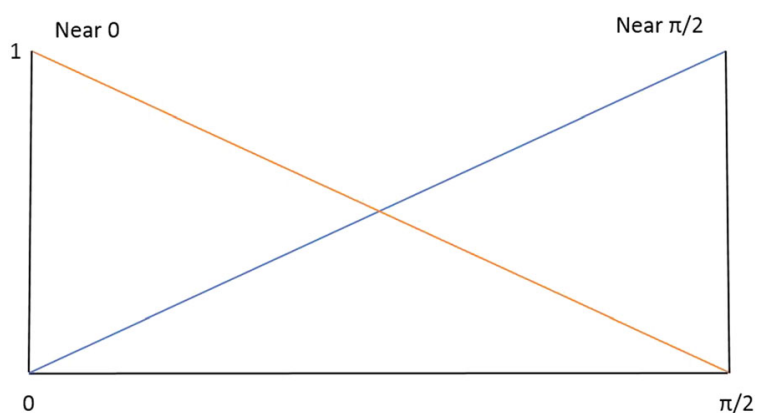

Figure 1. Membership functions of Rule 1 and Rule 2.

where

$A_{1}=\left[\begin{array}{cc}1 & T \\ \frac{g T}{4 L / 3-a m L} & 1\end{array}\right], \quad B_{1}=\left[\begin{array}{c}0 \\ -\frac{a T}{4 L / 3-a m L}\end{array}\right]$,

$F_{1}=\left[\begin{array}{l}\varepsilon_{1} T \\ \varepsilon_{2} T\end{array}\right]$,

$A_{2}=\left[\begin{array}{cc}1 & T \\ \frac{2 g T}{\pi\left(4 L / 3-a m L \delta^{2}\right)} & 1\end{array}\right]$,

$B_{1}=\left[\begin{array}{c}0 \\ -\frac{a \delta T}{4 L / 3-a m L \delta^{2}}\end{array}\right]$,

$F_{2}=\left[\begin{array}{l}\varepsilon_{1} T \\ \varepsilon_{2} T\end{array}\right]$ with $\delta=\cos \left(80^{\circ}\right)$.

The following values are used in our simulation:

$M=8.0 \mathrm{~kg}, \quad m=2 \mathrm{~kg}, \quad L=0.5 \mathrm{~m}, \quad g=9.8 \mathrm{~m} / \mathrm{s}^{2}$,

$\varepsilon_{1}=1, \quad \varepsilon_{2}=0$,

with the sampling time $T=0.001$ and initial conditions $x_{1}=\pi / 6, \quad x_{2}=-\pi / 6$. The membership of Rule 1 and Rule 2 is shown in Figure 1.

After the system discretization, the feedback control gain can be found from Equation (23) by solving the LMI (20) at each time step. The following design parameters are chosen to satisfy:

Mixed NLQR- $H_{\infty}$ criteria:

$C=\left[\begin{array}{ll}1 & 1\end{array}\right], \quad D=[1], \quad Q=\operatorname{diag}\left\{\left[\begin{array}{ll}80 & 1\end{array}\right]\right\}, \quad R=1$ ，

$\alpha=1 \quad \beta=0, \quad \gamma=-5$.

Mixed NLQR-passivity criteria:

$C=\left[\begin{array}{ll}1 & 1\end{array}\right], \quad D=[1], \quad Q=\operatorname{diag}\left\{\left[\begin{array}{ll}80 & 1\end{array}\right]\right\}, \quad R=1$,

$\alpha=1, \quad \beta=5, \quad \gamma=0$.

The mixed criteria control performance results are shown in Figures 2-4. From these figures, we find that the novel fuzzy LMI control has satisfactory performance. The new technique controls the inverted pendulum well under the effect of finite energy disturbance. 


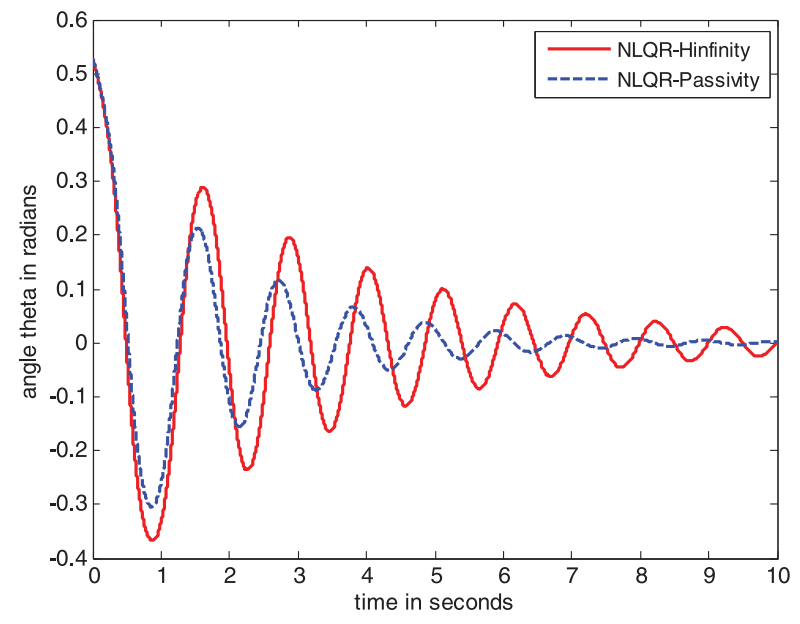

Figure 2. Angle trajectory of the inverted pendulum.

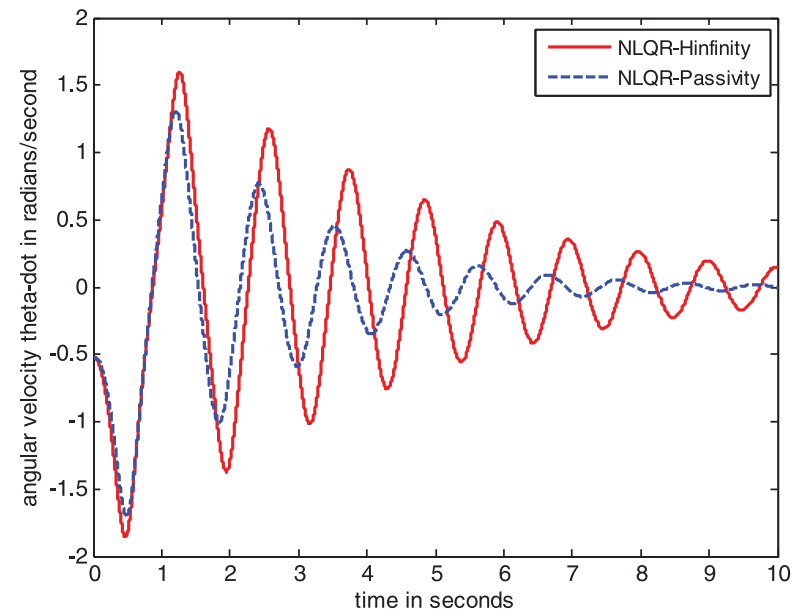

Figure 3. Angular velocity trajectory of the inverted pendulum.

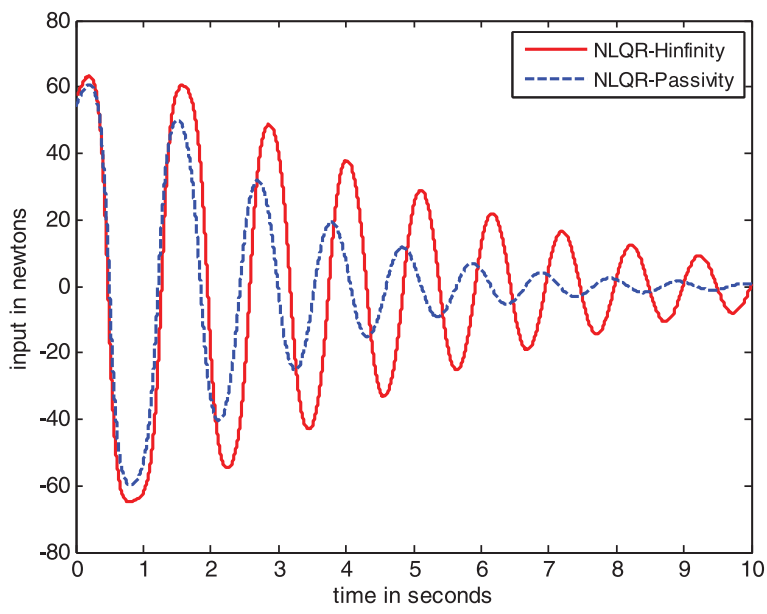

Figure 4. Control input applied to the inverted pendulum.

\section{Conclusions}

This paper presents a novel discrete-time nonlinear system fuzzy control approach based on the LMI solutions. We have first applied the Takagi-Sugeno fuzzy model to decompose the nonlinear system. Mixed performance criteria have been used to design the controller and the relative weighting matrices of these criteria can be achieved by choosing different coefficient matrices. The optimal control can be obtained by solving the LMI at each time step. The benchmark inverted pendulum on a cart problem has been used as an example to demonstrate its effectiveness. The simulation studies show that the proposed method provides a satisfactory alternative to the existing nonlinear control approaches.

\section{Disclosure statement}

No potential conflict of interest was reported by the authors.

\section{References}

Basar, T., \& Bernhard, P. (1995). H-infinity optimal control and related minimax design problems - Adynamic game approach (2nd ed.). Boston, MA: Birkhauser.

Baumann, W. T., \& Rugh, W. J. (1986). Feedback control of nonlinear systems by extended linearization. IEEE Transactions on Automatic Control, 31(1), 40-46.

Boyd, S., Ghaoui, L. E., Feron, E., \& Balakrishnan, V. (1994). Linear matrix inequalities in system and control theory. Philadelphia, PA: SIAM Studies in Applied Mathematics, SIAM.

Huang, Y., \& Lu, W-M. (1996). Nonlinear optimal control: Alternatives to Hamilton-Jacobi equation. Proceedings of 35th Conference on Decision and Control, Kobe, Japan, pp. 3942-3947.

Khalil, H. K. (2002). Nonlinear systems (3rd ed.). Upper Saddle River, NJ: Prentice Hall.

Mohseni, J., Yaz, E., \& Olejniczak, K. (1998). State dependent LMI control of discrete-time nonlinear systems. Proceedings. of the 37th IEEE Conference on Decision and Control, Tampa, FL, pp. 4626-4627.

Takagi, T., \& Sugeno, M. (1985). Fuzzy identification of systems and its applications to model and control. IEEE Transactions on Systems, Man, and Cybernetics, 15, 116-132.

Tanaka, K., Ikeda, T., \& Wang, H. O. (1996). Design of fuzzy control systems based on relaxed LMI stability conditions. 35th IEEE Conference on Decision and Control, Kobe, Vol. 1, pp. 598-603.

Tanaka, K., \& Sugeno, M. (1990). Stability analysis of fuzzy systems using Lyapunov's direct method. Proceedings NAFIPS'90, pp. 133-136.

Tanaka, K., \& Wang, H. O. (2001). Fuzzy control systems design and analysis-A linear matrix inequality approach. New York, NY: Wiley.

Van der Shaft, A. J. (1993). Nonlinear state space $H_{\infty}$ control theory. In H. J. Trentelman \& J. C. Willems (Eds.), Perspectives in control (pp. 153-190). Boston, MA: Birkhauser.

Vidyasagar, M. (2002). Nonlinear system analysis (2nd ed.). Englewood Cliffs, NJ: SIAM.

Wang, H. O., Tanaka, K., \& Griffin, M. (1996). An approach to fuzzy control of nonlinear systems: Stability and design issues. IEEE Transactions on Fuzzy Systems, 4(1), 14-23.

Wang, L. X. (1994). Adaptive fuzzy systems and control: Design and stability analysis. Englewood Cliffs, NJ: Prentice Hall.

Wang, X., \& Yaz, E. E. (2009). The state dependent control of continuous-time nonlinear systems with mixed performance criteria. Proceedings of IASTED International Conference 
on Identification Control and Applications, Honolulu, HI, pp. 98-102.

Wang, X., \& Yaz, E. E. (2010, July). Robust multi-criteria optimal fuzzy control of continuous-time nonlinear systems. Proceedings of the 2010 American Control Conference, Baltimore, MD, USA, pp. 6460-6465.

Wang, X., \& Yaz, E. E. (2010, December). Robust multi-criteria optimal fuzzy control of discrete-time nonlinear systems. Proceedings of the 49th IEEE Conference on Decision and Control, Atlanta, Georgia, USA, pp. 4269-4274.

Wang, X., Yaz, E. E., \& Jeong, C. S. (2010, July). Robust nonlinear feedback control of discrete-time nonlinear systems with mixed performance criteria. Proceedings of the 2010 American Control Conference, Baltimore, MD, USA, pp. 63576362.

Wang, X., Yaz, E. E., \& Yaz, Y. I. (2010, December). Robust and resilient state dependent control of continuous-time nonlinear systems with general performance criteria. Proceedings of the 49th IEEE Conference on Decision and Control, Atlanta, Georgia, USA, pp. 603-608.

Wang, X., Yaz, E. E., \& Yaz, Y. I. (2011). Robust and resilient state dependent control of discrete time nonlinear systems with general performance criteria, Proceedings of the 18th IFAC Congress, Milano, Italy, pp. 10904-10909. 